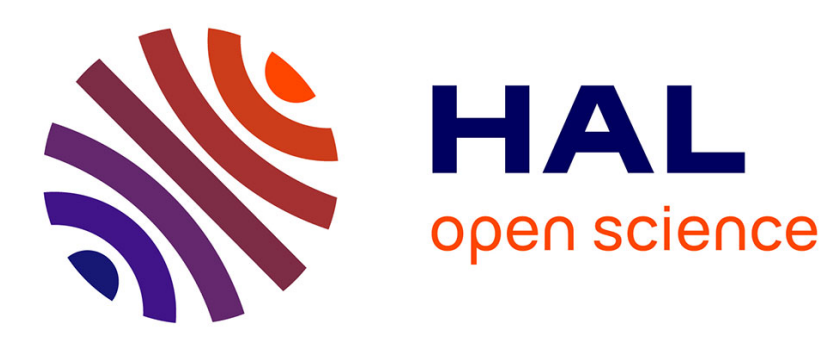

\title{
Comparing quadratic and non-quadratic local risk minimization for the hedging of contingent claims
}

\author{
Frédéric Abergel
}

\section{To cite this version:}

Frédéric Abergel. Comparing quadratic and non-quadratic local risk minimization for the hedging of contingent claims. 2013. hal-00771528

\section{HAL Id: hal-00771528 \\ https://hal.science/hal-00771528}

Preprint submitted on 8 Jan 2013

HAL is a multi-disciplinary open access archive for the deposit and dissemination of scientific research documents, whether they are published or not. The documents may come from teaching and research institutions in France or abroad, or from public or private research centers.
L'archive ouverte pluridisciplinaire HAL, est destinée au dépôt et à la diffusion de documents scientifiques de niveau recherche, publiés ou non, émanant des établissements d'enseignement et de recherche français ou étrangers, des laboratoires publics ou privés. 


\title{
Comparing quadratic and non-quadratic local risk minimization for the hedging of contingent claims
}

\author{
Frederic Abergel, \\ Chair of Quantitative Finance \\ Laboratory of Mathematics Applied to Systems \\ École Centrale Paris \\ 92290 Châtenay Malabry \\ France
}

\begin{abstract}
In this note, I study further the approach introduced in [1] for the hedging of derivatives in incomplete markets via local risk minimization. A structure result is provided, which essentially shows the equivalence between non-quadratic risk minimization under the historical probability and quadratic local risk minimization under an equivalent, implicitly defined probability.
\end{abstract}

\section{Introduction}

First introduced in the seminal work of [4], the quadratic local risk minimization approach to derivative hedging is well understood, and its connection to pricing under the minimal martingale measure is well established, see [3] [5] for detailed results. More recently, [1][2], a theory using non-quadratic functions to measure local risk has been presented. In this theory, a modified cost process is naturally introduced as the limit of a nonlinear, discrete stochastic integral. The Ito differential of this new cost process depends in a nonlinear fashion on that of the standard cost process $d C_{t}=d V_{t}-\delta_{t} d S_{t}$, and this nonlinear dependance leads to a nonlinear equation for the portfolio value process $V_{t}$ as well as a (possibly) nonlinear relationship between the price process and the hedging strategy $\delta$. Results are more accurate and better understood in the markovian case, where the asset $S$ is given as the solution of a stochastic differential equation, and this is the setting that prevails in the rest of this paper.

The paper is organized as follows: Section 1 consists in a short reminder of the notations and results in [1, where a general local risk function $f$ is introduced; then, the relationship between the $f$-cost and the classical cost of a strategy is worked out in Section 2, where a new probability measure appears in a natural fashion. In Section 3, the identity between the general local risk minimizing strategy and the quadratic local risk minimizing strategy under this new probability measure is proven. Finally, Section 4 is devoted to a reformulation of the optimality condition as a fixed-point problem for the portfolio value process.

\section{Some notations and earlier results}

The key notations and concepts of [1] are now recalled for the sake of completeness.

$(\Omega, \mathcal{F}, P)$ is a probability space with a filtration $\left(\mathcal{F}_{t}\right)_{0 \leq t \leq T}$ satisfying the usual conditions of right-continuity and completeness. $T \in \mathbb{R}^{*+}$ denotes a fixed and finite time horizon. Furthermore, $\mathcal{F}_{0}$ is trivial and $\mathcal{F}_{T}=\mathcal{F}$. The tradablee, risky asset $S=\left(S_{t}\right)_{0 \leq t \leq T}$ is a strictly positive, continuous semimartingale

$$
S=S_{0}+N+A
$$


such that $N=\left(N_{t}\right)_{0 \leq t \leq T}$ is a continuous square-integrable martingale with $N_{0}=0$, and $A=\left(A_{t}\right)_{0 \leq t \leq T}$ is a continuous and adapted process of finite variation $|A|$ with $A_{0}=0$.

A general trading strategy $\Phi$ consists in a pair of càdlàg and adapted processes $\delta=\left(\delta_{t}\right)_{0 \leq t \leq T}$ and $\beta=$ $\left(\beta_{t}\right)_{0 \leq t \leq T}$, while a contingent claim is described by a random variable $H \in L^{2}(P)$ with $H=\delta^{H} S_{T}+\beta^{H}$, $\delta^{H}$ and $\beta^{H}$ being $\mathcal{F}_{T}$-measurable random variables. Associated to any trading strategy is the value process $V=\delta S+\beta$.

Next are two important definitions:

Definition 1.0.1 A trading strategy will be called $H$-admissible if it meets the following requirements

$$
\left\{\begin{array}{l}
\delta_{T}=\delta^{H} P-\text { a.s. } \\
\beta_{T}=\beta^{H} P-\text { a.s. } \\
\delta \text { has finite and integrable quadratic variation } \\
\beta \text { has finite and integrable quadratic variation } \\
\delta \text { and } \beta \text { have finite and integrable quadratic covariation. }
\end{array}\right.
$$

Definition 1.0.2 For an $H$-admissible strategy $\Phi$, the associated $f$-cost process ${ }^{f} C_{t}(\Phi)$ is defined as the following limit

$$
\lim _{n \rightarrow \infty} \sum_{k=1}^{l_{n}} f^{\prime}\left(\beta^{\tau_{k}^{n}}-\beta^{\tau_{k-1}^{n}}+\left(\delta^{\tau_{k}^{n}}-\delta^{\tau_{k-1}^{n}}\right) S^{\tau_{k}^{n}}\right)
$$

whenever it exists.

Here, convergence is required in ucp topology for any sequences $\mathcal{P}_{n}$ of Riemann partitions of $[0, T]$ of length $l_{n}$, and we have used the notation $X^{T}$ for the process stopped at $T$.

A general result concerning the $f$-cost process is the

Theorem 1.1 [1] The $f$-cost process of an $H$-admissible strategy $\Phi$ can be expressed in terms of the portfolio value $V$

$$
\begin{gathered}
{ }^{f} C_{t}(\Phi)= \\
f^{\prime \prime}(0)\left(V_{t}-V_{0}-\int_{0+}^{t} \delta_{s-} d S_{s}\right) \\
+\frac{f^{(3)}(0)}{2}\left([V, V]_{t}^{c}-2 \int_{0+}^{t} \delta_{s-} d[V, S]_{s}^{c}+\int_{0+}^{t} \delta_{s-}^{2} d[S, S]_{s}^{c}\right) \\
+\sum_{0<s \leq t} f^{\prime}\left(\Delta V_{s}-\delta_{s-} \Delta S_{s}\right)-f^{\prime \prime}(0)\left(\Delta V_{s}-\delta_{s-} \Delta S_{s}\right)
\end{gathered}
$$

with notation $[X, Y]^{c}$ (see [6]) standing for the continuous part of the (càdlàg) quadratic covariation process.

In a nutshell, the theory in [1] states that a minimizing strategy associated to the risk function $f$ is characterized by a continuous martingale $M_{t}$ orthogonal to the (martingale part of the) price process $S_{t}$, such that there holds

$$
{ }^{f} C_{t}=M_{t} .
$$

Note that 1.2 actually characterizes those strategies that are pseudo-optimal in the terminology of [1], see [2] for an in-depth discussion of the relation between local risk minimizing and pseudo-optimal strategies. Suffice it to say that, in the context of processes modelled by stochastic differential equations, both notions can be proven to be identical, and any possible difference will be overlooked in the rest of this paper. 


\section{Comparing the $f$-cost and the usual cost}

Using Theorem 1.1, one easily shows the

Proposition 2.1 Under the assumption that the process $S$ is continuous, the $f$-cost process and the usual cost process are related to one another by

$$
{ }^{f} C_{t}=f^{\prime \prime}(0) C_{t}+\frac{f^{(3)}(0)}{2}<C, C>_{t}
$$

Here, $C \equiv \frac{x^{2}}{2} C$ denotes, with a slight abuse of notation, the cost process associated to the quadratic risk function $x \rightarrow \frac{x^{2}}{2}$.

For simplicity, the function $f$ will be assumed to be normalized in order that $f$ " $(0)=1$. Now, the optimality condition $(1.2$ can be rewritten in terms of the usual cost process as

$$
C_{t}+\lambda<C, C>_{t}=M_{t}
$$

(with $\left.\frac{f^{(3)}(0)}{2}=\lambda\right)$, which is obviously equivalent to

$$
C_{t}=M_{t}-\lambda<M, M>_{t} .
$$

Upon introducing the Doleans-Dade exponential $\mathcal{E}(\lambda M)$ of $\lambda M$, solution on $(0, T)$ to

$$
d \phi_{t}=\lambda \phi_{t} d M_{t},
$$

and defining an absolutely continuous change of probability measure by its conditional density

$$
\frac{d \mathcal{Q}}{d \mathcal{P}} \mid \mathcal{F}_{t}=\phi_{t}
$$

one can rephrase 2.3 by saying that $C$ is a $\mathcal{Q}$-martingale orthogonal to $S$.

Note that $\mathcal{Q}$ is precisely the minimal martingale measure associated to the cost process $C$ as defined in [5], see also Theorem 1 in [3] where the role of the structure assumption (1.2) is played by (2.3).

It is known, see 3, that the quadratic local risk minimizing strategy generates a cost process that is a martingale orthogonal to $S$, and a value process that has the representation $V_{t}=E_{\mathcal{P} *}\left(H \mid \mathcal{F}_{t}\right)$ under the minimal martingale measure associated to the process $S$. One is therefore led to address a very natural question: is there a relation between the local risk minimizing strategy associated to $f$ under $\mathcal{P}$, and the quadratic local risk minimizing strategy under the new probability $\mathcal{Q}$ ?

A positive answer to that question is provided in the next section.

\section{An equivalent quadratic problem}

For the sake of clarity, let us first work out the case of a simple stochastic volatility model driven by a two-dimensional standard Wiener process $\left(W_{t}^{1}, W_{t}^{2}\right)$

$$
\begin{aligned}
& \frac{d S_{t}}{S_{t}}=F\left(S_{t}, \sigma_{t}, t\right) d W_{t}^{1}+\mu\left(S_{t}, \sigma_{t}, t\right) d t \\
& d \sigma_{t}=\alpha\left(S_{t}, \sigma_{t}, t\right) d W_{t}^{2}+\beta\left(S_{t}, \sigma_{t}, t\right) d t
\end{aligned}
$$

with smooth enough coefficients $F, \mu, \alpha, \beta$.

In this context, see Section 4 in [1], the $f$-cost process associated to the optimal strategy is given by

$$
{ }^{f} C_{t}=\int_{0}^{t} \alpha \frac{\partial V}{\partial \sigma} d W_{s}^{2},
$$


where the value process $V$ is solution to the equation

$$
\mathcal{L}_{\mathcal{P}} V+\lambda \alpha^{2}\left(\frac{\partial V}{\partial \sigma}\right)^{2}=0
$$

with $\mathcal{L}_{\mathcal{P}} \psi \equiv \frac{1}{2} F^{2} S^{2} \frac{\partial^{2} \psi}{\partial S^{2}}+\frac{1}{2} \alpha^{2} \frac{\partial^{2} \psi}{\partial \sigma^{2}}+\beta \frac{\partial \psi}{\partial \sigma}$.

Under the new measure defined by (2.5) with

$$
M_{t}=\int_{0}^{t} \alpha \frac{\partial V}{\partial \sigma} d W_{s}^{2}
$$

the infinitesimal generator $\mathcal{L}_{\mathcal{P}}$ changes to

$$
\mathcal{L}_{\mathcal{Q}} \equiv \mathcal{L}_{\mathcal{P}}+\left(\lambda \alpha^{2} \frac{\partial V}{\partial \sigma}\right) \frac{\partial}{\partial \sigma} .
$$

Consider now that $\mathcal{Q}$ is the reference probability: the optimal strategy associated to the quadratic local risk minimization under $\mathcal{Q}$ generates a cost process $\tilde{C}$ such that

$$
\tilde{C}_{t}=\int_{0}^{t} \alpha \frac{\partial \tilde{V}}{\partial \sigma} d \tilde{W}_{s}^{2}
$$

where $\tilde{W}_{t}^{2} \equiv W_{t}^{2}-\int_{0}^{t} \lambda \alpha \frac{\partial V}{\partial \sigma} d s$ is a standard Wiener process under $\mathcal{Q}$ and the new value process $\tilde{V}$ is given by the solution to

$$
\mathcal{L}_{\mathcal{Q}} \tilde{V}=0
$$

Now, it is trivial to remark that $\mathrm{V}$, being a solution to (3.4), also solves 3.8. Since uniqueness holds for the linear $\mathrm{PDE}(3.8), \tilde{V} \equiv V$ is its only solution. Hence, the general local risk minimization problem can naturally be recast into a quadratic one, up to a change of probability measure.

This result can easily be generalized to a market driven by a $(K+D)$-dimensional Wiener process corresponding to $K$ tradable assets with $D$ volatility-like "noises". In fact, there holds the

Theorem 3.1 Consider the $(K+D)$-dimensional process $\left(S^{1}, \ldots, S^{K}, y^{K+1}, \ldots, y^{K+D}\right)$ solution to the $(K+$ $D$-dimensional system of stochastic differential equations:

for $i=1, \ldots, K$

$$
\frac{d S_{t}^{i}}{S_{t}^{i}}=F\left(S_{t}^{1}, \ldots, S_{t}^{K}, y_{t}^{K+1}, \ldots, y_{t}^{K+D}, t\right) d W_{t}^{i}+\mu^{i}\left(S_{t}^{1}, \ldots, S_{t}^{K}, y_{t}^{K+1}, \ldots, y_{t}^{K+D}, t\right) d t
$$

for $i=K+1, \ldots, K+D$

$$
d y_{t}^{i}=\alpha_{i}\left(S_{t}^{1}, \ldots, S_{t}^{K}, y_{t}^{K+1}, \ldots, y_{t}^{K+D}, t\right) d W_{t}^{i}+\beta_{i}\left(S_{t}^{1}, \ldots, S_{t}^{K}, y_{t}^{K+1}, \ldots, y_{t}^{K+D}, t\right) d t .
$$

Then, the optimal strategy for the local risk minimization with risk function $f$ also solves the quadratic local risk minimization problem under the new measure defined by 2.5, with this time

$$
M_{t}=\sum_{i=K+1}^{K+D} \int_{0}^{t} \alpha^{i} \frac{\partial V}{\partial y^{i}} d W_{s}^{i} .
$$

Proof: Proposition 3.1 is obviously an extension of the simple example treated above in the case $K=D=1$. Upon applying the change of probability 2.5 and computing

$$
E\left(\phi_{t+h} f\left(S_{t+h}^{1}, \ldots, S_{t+h}^{K}, y_{t+h}^{K+1}, \ldots, y_{t+h}^{K+D}, t+h\right) \mid \mathcal{F}_{t}\right),
$$


one shows that, under the new probability $\mathcal{Q}$, the infinitesimal generator of the diffusion is transformed according to the following rule:

$$
\left(\mathcal{L}_{\mathcal{Q}} f\right)\left(S_{t}^{1}, \ldots, S_{t}^{K}, y_{t}^{K+1}, \ldots, y_{t}^{K+D}, t\right)=\left(\mathcal{L}_{\mathcal{P}} f\right)\left(S_{t}^{1}, \ldots, S_{t}^{K}, y_{t}^{K+1}, \ldots, y_{t}^{K+D}, t\right)+\lambda \sum_{i=K+1}^{K+D}\left(\alpha^{i}\right)^{2} \frac{\partial V}{\partial y^{i}} \frac{\partial}{\partial y^{i}}
$$

Then, the argument goes exactly as that outlined in the case $K=D=1$ : the partial differential equation for the value $\tilde{V}$ of the optimal portfolio in the case of a quadratic local risk minimizing strategy under $\mathcal{Q}$ is precisely

$$
\mathcal{L}_{\mathcal{Q}} \tilde{V}=0
$$

for which a solution - actually, the solution, thanks to the uniqueness property for linear parabolic equations - is already known and given by $V$, the optimal portfolio value for the local risk associated to $f$. Moreover, the optimal cost process under the new probability $\mathcal{Q}$ is precisely equal to $\sum_{i=K+1}^{K+D} \int_{0}^{t} \alpha^{i} \frac{\partial V}{\partial y^{i}} d \tilde{W}_{s}^{i}$, where $\left(\tilde{W}^{i}\right)$ is a standard $D$-dimensional Wiener process under $\mathcal{Q}$.

Finally, note that the case of a correlated $(K+D)$-dimensional Wiener process is dealt with in a similar fashion, with computations that are slightly more involved.

\section{A fixed-point formulation for the value process}

In this section, we make use of the representation formula for the optimal value process in the quadratic case as an expectation under the minimal martingale measure, see [5].

In view of this representation formula, the value process $V$ provided by Theorem 3.1 can be obtained via two successive changes of measures: a change from $\mathcal{P}$ to $\mathcal{Q}$ with conditional density

$$
\frac{d \mathcal{Q}}{d \mathcal{P}} \mid \mathcal{F}_{t}=\mathcal{E}\left(\lambda M_{t}\right)
$$

followed by a change of measure from $\mathcal{Q}$ to the minimal martingale measure of the price process $S, \mathcal{Q}^{*}$, say. This procedure yields an expression for the value process $V$ as a conditional expectation under $\mathcal{Q}^{*}$

$$
V_{t}=E_{\mathcal{Q}^{*}}\left(H \mid \mathcal{F}_{t}\right) .
$$

One can work out the two changes of measure in the simple example at the beginning of Section 3 and obtain

$$
V_{t}=E_{\mathcal{P}}\left(\mathcal{E}\left(\lambda \int_{0}^{t} \alpha \frac{\partial V}{\partial \sigma} d W_{s}^{2}-\int_{0}^{t} \frac{\mu}{\sigma} d W_{s}^{1}\right) H \mid \mathcal{F}_{t}\right) .
$$

Equation 4.3 can be used as a defining equation for the value process $V$ via a fixed point argument. Obviously, this new theory is not as satisfactory as in the quadratic case, in the sense that the change of measure depends on the claim itself through the value process.

\section{References}

[1] F. Abergel, N. Millot, Non quadratic local risk minimization for hedging contingent claims in incomplete markets, SIAM J. Finan. Math. 2-1 (2011), pp. 342-356

[2] N. Millot, PhD thesis, École Centrale Paris, 2012

[3] M. Schweizer, On the minimal martingale measure and the Follmer-Schweizer decomposition, Stochastic Analysis and Applications 13 (1995), 573-599

[4] H. Follmer, M. Schweizer, Hedging by sequential regression: an introduction to the mathematics of option trading, ASTIN Bulletin (1985), vol. 19 
[5] H. Follmer, M. Schweizer, Hedging of contingent claims under incomplete information, in M. H. A. Davis, R. J. Elliott, editors, Applied Stochastic Analysis, Stochastics Monographs, vol. 5, Gordon and Breach, London/New York (1991), 389-414

[6] P. Protter, Stochastic integration and differential equations, Applications of Mathematics, Springer, 1995 